\title{
Nondestructive extraction of junction depths of active doping profiles from photomodulated optical reflectance offset curves
}

\author{
Janusz Bogdanowicz ${ }^{\mathrm{a})}$ \\ Instituut voor Kern-en Stralingsfysika, KU Leuven, Celestijnenlaan 200D B-3001 Leuven, Belgium \\ and IMEC, Kapeldreef 75, B-3001 Leuven, Belgium \\ Fabian Dortu ${ }^{\text {b) }}$ and Trudo Clarysse \\ IMEC, Kapeldreef 75, B-3001 Leuven, Belgium \\ Wilfried Vandervorst \\ Instituut voor Kern-en Stralingsfysika, KU Leuven, Celestijnenlaan 200D, B-3001 Leuven, Belgium \\ and IMEC, Kapeldreef 75, B-3001 Leuven, Belgium
}

Erik Rosseel and Ngoc Duy Nguyen

IMEC, Kapeldreef 75, B-3001 Leuven, Belgium

Derrick Shaughnessy, Alex Salnik, and Lena Nicolaides

KLA-Tencor Corp., 160 Rio Robles, San Jose, California 95134

(Received 12 June 2009; accepted 2 November 2009; published 1 March 2010)

\begin{abstract}
The ITRS Roadmap highlights the electrical characterization of the source and drain extension regions as a key challenge for future complimentary-metal-oxide-semiconductor technology. Presently, an accurate determination of the depth of ultrashallow junctions can routinely only be performed by time-consuming and destructive techniques such as secondary ion mass spectrometry (SIMS). In this work, the authors propose to use the fast and nondestructive photomodulated optical reflectance (PMOR) technique, as implemented in the Therma-Probe ${ }^{\circledR}$ (TP) dopant metrology system, for these purposes. PMOR is a pump-probe technique based on the measurement of the pump-induced modulated change in probe reflectance, i.e., the so-called (photo) modulated reflectance. In this article, the authors demonstrate that the absolute junction depths of boxlike active dopant structures can be extracted in a very simple and straightforward way from the TP offset curves, which represent the behavior of the modulated reflectance as a function of the pump-probe beam spacing. Although the procedure is based on the insights into the physical behavior of the offset curves, no modeling is involved in the actual extraction process itself. The extracted junction depths are in good correlation with the corresponding junction depths as measured by means of SIMS. The technique has a subnanometer depth sensitivity for depths ranging from 10 to $35 \mathrm{~nm}$ with the present Therma-Probe ${ }^{\circledR}$ 630XP system. The extension of the proposed procedure to the general ultrashallow profiles is also explored and discussed. () 2010 American Vacuum Society. [DOI: 10.1116/1.3269737]
\end{abstract}

\section{INTRODUCTION}

The electrical characterization of ultrashallow junctions (USJs) such as the source and drain extension regions of a metal-oxide-semiconductor transistor is regarded by the ITRS Roadmap ${ }^{1}$ as one of the most critical challenges for future technology nodes. To solve this problem, one usually relies on secondary ion mass spectrometry (SIMS) for the determination of the junction depth and to four-point probe for the extraction of the sheet resistance and therefore of the activation level of the doping profile. However, this combination of techniques, besides being quite time consuming, suffers from significant drawbacks in their applications to shallow USJs., ${ }^{2,3}$

The photomodulated optical reflectance $(\mathrm{PMOR})^{4-9}$ has been demonstrated in the past to be a promising technique for USJ parameter characterization. PMOR is a contactless

\footnotetext{
a)Electronic addresses: janusz.bogdanowicz@imec.be and janusz@imec.be

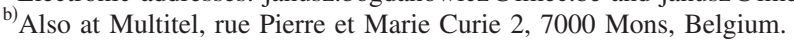

pump-probe beam technique that records the time-dependent changes in probe reflectance due to the pump-induced increase in excess carrier concentration ${ }^{10}$ and in surface temperature. ${ }^{11}$ This article discusses experiments performed with the Therma-Probe ${ }^{\circledR}(\mathrm{TP})^{12,13}$ system, which is an implementation of PMOR with high pump power modulation frequency $(1 \mathrm{MHz})$, with highly localized pump and probe beam spots $($ radii $=0.5 \mu \mathrm{m}$ ). The pump and probe beam intensities in TP are, respectively, 13.5 and $2.5 \mathrm{~mW}$. The respective wavelengths are 790 and $670 \mathrm{~nm}$. We have already shown the capability of the TP system to reconstruct uniquely boxlike carrier profiles based on using the two independent signals, ${ }^{12,14,15}$ respectively, in-phase $(I)$ and inquadrature $(Q)$ with the pump power. However, it seems that the range of variations in $Q$ is so close to the noise level that this technique is hardly usable with the laser settings of the current TP630XP tool.

In this article, we use the PMOR signals measured with the pump and probe beams separated by a few micrometers 
(offset distance) to determine the junction depth of active doping profiles. In Sec. II, a basic theory of the PMOR signal generation from boxlike active doping profiles, including their lateral behavior, is briefly described. Based on this theory and on the experimental data, we propose a procedure to extract the junction depth of boxlike profiles in Sec. III. In Sec. IV, we show that the technique can be generalized to more complex active doping profiles such as annealed implanted profiles. Finally, in Sec. V, we show that when implemented on the TP630XP dopant metrology system, the technique has a subnanometer depth sensitivity for junction depths ranging from 10 to $35 \mathrm{~nm}$.

\section{THEORY OF PMOR ON ACTIVE DOPING PROFILES}

In this section, we very briefly summarize how to derive an approximate formula to describe the behavior of the PMOR signals on boxlike active doping profiles. We then generalize the theory to account for the lateral behavior of the signal, i.e., when the pump and the probe lasers are separated.

As explained in Sec. I, PMOR is an optical technique using two lasers. First, the pump laser, having a photon energy higher than the semiconductor bandgap, generates not only an excess temperature distribution but also an excess carrier distribution. Since the pump power is modulated, both the excess carrier concentration and the surface temperature vary with time. Second, the probe laser is reflected off the sample and the time-dependent variation in probe reflection (i.e., due to the pump laser) is coupled from the photodetector to a lock-in amplifier, which filters out the PMOR signal with very high sensitivity. In the case of a boxlike active doping profile with junction depth $X_{j}$ and active doping concentration $N_{\text {active }}$, the PMOR signal $\Delta \mathrm{R} / \mathrm{R}$ is shown $^{16,17}$ to vary as

$$
\begin{aligned}
\frac{\Delta R}{R}= & \frac{4}{n_{0}^{2}-1}\left\{-\frac{|\beta|}{m_{e}+m_{h}}[\underbrace{\left(1-\cos \left(\frac{4 \pi n_{0} X_{j}}{\lambda}\right)\right) \frac{\Delta N_{\text {substrate }}^{2}}{N_{\text {active }}}}_{\text {layer }}\right. \\
& +\underbrace{\cos \left(\frac{4 \pi n_{0} X_{j}}{\lambda}\right) \Delta N_{\text {substrate }}}_{\text {substrate }}]+\underbrace{\delta \Delta T_{\text {surface }}}_{\text {temperaturc }}\}
\end{aligned}
$$

where $m_{e}$ and $m_{h}$ are, respectively, the electron and hole effective masses, $n_{0}$ is the intrinsic silicon lattice refractive index at the probe wavelength $\lambda, \beta=-m \partial n / \partial N$ is the Drude coefficient $^{10}$ accounting for the variations in the refractive index $n$ due to the presence of excess free carriers of effective mass $m$, and $\delta=\partial n / \partial T$ accounts for the variations in refractive index due to the temperature rise. ${ }^{11} \Delta T_{\text {surface }}$ is the excess temperature at the surface, $\Delta N_{\text {surface }}$ is the excess carrier concentration in the substrate, $\Delta N_{\text {substrate }}^{2} / N_{\text {active }}$ is the excess carrier concentration in the layer under the following four assumptions. We need to assume, first, Boltzmann statistics. The second assumption is that there is no bandgap narrowing. Third, the quasi-Fermi levels are flat through the space-charge regions, and finally, $\Delta N_{\text {substrate }}<N_{\text {active- }}{ }^{16,17}$ Formula (1) shows that the signal is composed of three components related, first, to the excess carrier concentration in the layer (layer plasma component), second, to the excess carrier concentration in the substrate (substrate plasma component), and third, to the excess temperature (thermal component). Interestingly, formula (1) also demonstrates directly how PMOR is sensitive to the doping concentration and the junction depth.

Formula (1) predicts the behavior of the PMOR signal when the probe and the pump lasers are coincident. However, the purpose of this article is to study the lateral behavior of the PMOR signal, i.e., when the two laser beams are separated. We can, as a first approximation, assume that the layer plasma component, the substrate plasma component, and the thermal component all decay exponentially with respective decay lengths $L_{\text {layer }}, L_{\text {substrate, }}$ and $L_{\text {thermal }}$. If $x$ is the lateral distance between the pump and the probe beams, the total signal has the following behavior:

$$
\begin{aligned}
\frac{\Delta R}{R}(x)= & \frac{4}{n_{0}^{2}-1}\left\{-\frac{|\beta|}{m_{e}+m_{h}}\left[\left(1-\cos \left(\frac{4 \pi n_{0} X_{j}}{\lambda}\right)\right)\right.\right. \\
& \times \frac{\Delta N_{0, \text { substrate }}^{2}}{N_{\text {active }}} \exp \left(-x / L_{\text {layer }}\right) \\
& \left.+\cos \left(\frac{4 \pi n_{0} X_{j}}{\lambda}\right) \Delta N_{0, \text { substrate }} \exp \left(-x / L_{\text {substrate }}\right)\right] \\
& \left.+\delta \Delta T_{0, \text { surface }} \exp \left(-x / L_{\text {thermal }}\right)\right\}
\end{aligned}
$$

where $\Delta N_{0 \text {,substrate }}$ and $\Delta T_{0 \text {,surface }}$ are the substrate excess carrier concentration and excess temperature at $x=0$, i.e., when the two laser spots are coincident. The three involved decay lengths have different values. First, it is known ${ }^{18}$ that in a one-dimensional and low modulation frequency problem, $L_{\text {substrate }}=\sqrt{D \tau}$ and $L_{\text {thermal }}=\sqrt{2 D_{\text {th }} / \omega_{\text {pump }}}$, where $D$ is the carrier diffusivity, $\tau$ is the carrier lifetime, $D_{\text {th }}$ is the thermal diffusivity of the sample, and $\omega_{\text {pump }}$ is the pump angular modulation frequency. For TP, this typically gives $L_{\text {substrate }}$ $\sim 10 \mu \mathrm{m}$ and $L_{\text {thermal }} \sim 4 \mu \mathrm{m}$ in Si. Second, it can be shown that the decay length $L_{\text {layer }}$ of the layer plasma component is also shorter than $L_{\text {substrate }}{ }^{19}$ In other words, the thermal component and layer plasma components decay faster than the substrate plasma component. Notice that the given values are only valid in the one-dimensional case, they are actually shorter in a three-dimensional problem. In conclusion, we can claim that when the distance between the two lasers is large enough, only the substrate plasma component remains, i.e.,

$$
\begin{aligned}
\frac{\Delta R}{R} \rightarrow & -\frac{4}{n_{0}^{2}-1} \frac{|\beta|}{m_{e}+m_{h}} \cos \left(\frac{4 \pi n_{0} X_{j}}{\lambda}\right) \Delta N_{0, \text { substrate }} \\
& \times \exp \left(-x / L_{\text {substrate }}\right) .
\end{aligned}
$$

For the sake of completeness, a final remark needs to be made concerning the time dependence of the three signal 
components. In TP, where the modulation frequency is relatively high $\left(\omega_{\text {pump }} \tau \sim 1\right)$, all three components have not only a real part but also an imaginary part to account for their time delay with respect to the pump power variations. In this case, only the in-phase signal behaves as shown by formula (3), while the full time dependence needs to be taken into account to explain the lateral behavior of $Q .{ }^{17}$ As a consequence, only the in-phase signal is used in the rest of this article.

\section{JUNCTION DEPTHS OF BOXLIKE PROFILES}

In this section, we use the above theory to derive a procedure for the extraction of the junction depth of a chemical vapor deposition (CVD) boxlike active doping profile as obtained in boron-doped epitaxial $\mathrm{Si}$ epilayers grown by means of CVD. We compare the obtained results with the corresponding SIMS junction depths. A similar procedure is then explained to measure the relative variations in the junction depth, e.g., over a whole wafer.

\section{A. Absolute determination of junction depths}

The main result of the previous section is summarized in formula (3). This formula is also valid for an undoped $\mathrm{Si}$ substrate; if one takes $X_{j}=0$, the ratio of formula (3) taken on a boxlike doping profile to that of an undoped substrate is

$$
\frac{\Delta R / R_{\text {layer }}^{\mathrm{I}}}{\Delta R / R_{\text {substrate }}^{\mathrm{I}}} \rightarrow \cos \left(\frac{4 \pi n_{0} X_{j}}{\lambda}\right)
$$

when the beam spacing is large enough. In Eq. (4), the excess carrier concentration in the substrate and its decay length are assumed to be independent of the doped layer, which is correct if the inactive doping is not too high. ${ }^{16}$ The meaning of formula (4) is that the ratio of the signals measured, respectively, on the studied sample and on an undoped substrate should give access to the junction depth of the sample if the pump and probe beams are sufficiently spaced apart. Keep in mind that, as explained at the end of Sec. II, this is only valid for the in-phase signal.

Let us apply this technique to the so-called CVD2 and CVD3 matrices as well as the CVD8 matrix. The CVD2 and CVD3 matrices are described in Table I of Ref. 12. The CVD8 matrix is composed of two series of samples. The CVD8-1 series consists of six single CVD layers with almost equal junction depths $(\sim 40 \mathrm{~nm})$ and different SIMS doping concentrations ranging from $10^{19}$ to $3 \times 10^{20} \mathrm{~cm}^{-3}$. The CVD8-2 series is composed of double box structures with the same junction depths (12 and $25 \mathrm{~nm}$, respectively, for the shallow and deep layers), same doping concentration in the shallow layer $\left(3 \times 10^{20} \mathrm{~cm}^{-3}\right)$, and varying doping concentrations in the second layer ranging from $3 \times 10^{19}$ to 3 $\times 10^{20} \mathrm{~cm}^{-3}$. All measurement data were obtained on a special TP system optimized for IMEC research needs with a maximum beam offset of $4 \mu \mathrm{m}$.

Figure 1 shows the offset behavior of the ratio of the TP signals measured in a selection of layers of the CVD2 matrix to the signals on an undoped substrate. In a large majority of

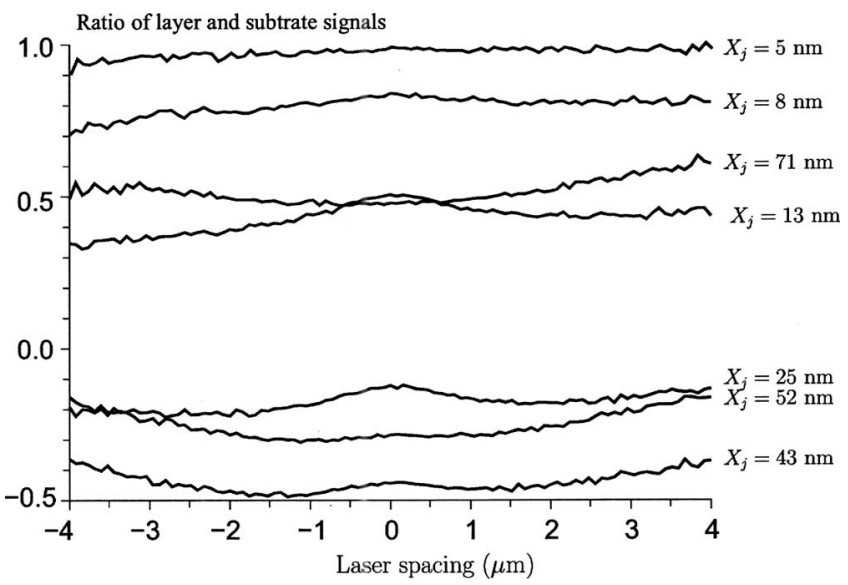

FIG. 1. Offset behavior of $\Delta R / R_{\text {layer }}^{\mathrm{I}} / \Delta R / R_{\text {substrate }}^{\mathrm{I}}$ on a selection of samples of the CVD2 matrix. At a $4 \mu \mathrm{m}$ separation, the saturation value can be used to determine the junction depth using formula (4). The SIMS junction depth is indicated on the right.

the cases (essentially for ultrashallow samples), the ratio saturates at constant values between -1 and 1 , as it should according to formula (4). This shows that the assumptions needed to derive the theory are valid. It is typically for the deepest junctions that the saturation is not reached, most likely due to the larger impact of inactive dopants on the substrate injection level ${ }^{16}$ (higher inactive dose).

Notice the unexpected asymmetry of the curves in Fig. 1, which is usually observed on active doping profiles in Si. This asymmetry can be explained by the introduction of carriers into the native oxide (charging) during the measurement, ${ }^{19,20}$ which passivates the surface. In Sec. V, we show that this effect can be taken advantage of in order to reduce the signal sensitivity to the surface conditions, making the above developed model more realistic. The reproducibility and accuracy are further enhanced in this case. Since these offset curves are measured from left to right, the surface is more charged while measuring the right part of the curve (spacing $>0$ ). Hence, the right part of these curves is systematically used throughout this article.

Figure 2 compares the junction depths obtained using formula (4) at $4 \mu \mathrm{m}$ laser beam separation to the measured SIMS junction depths. The correlation is very good for all ultrashallow samples $\left(X_{j}<30 \mathrm{~nm}\right)$ even for the double boxes of CVD8-2 series (correlation with the deeper junction). The discrepancies can be explained either by the junction depth nonuniformity on the wafers (SIMS profile is measured at the center, TP is measured as close as possible to the center) and by the substrate signal reproducibility (see Sec. V). The correlation for deeper junctions is not as good as could be expected by the nonsaturation in Fig. 1. This indeed shows that these samples do not perfectly comply with the developed theory. Notice also that due to the periodicity of formula (4), the technique cannot distinguish between a junction depth $X_{j}$ and $\left(\lambda / 2 n_{0}-X_{j}\right)$. In Fig. 2, we have therefore used the known SIMS junction depth to decide which formula to apply (see the two different zones separated by the $X_{j}=44 \mathrm{~nm}$ line). 


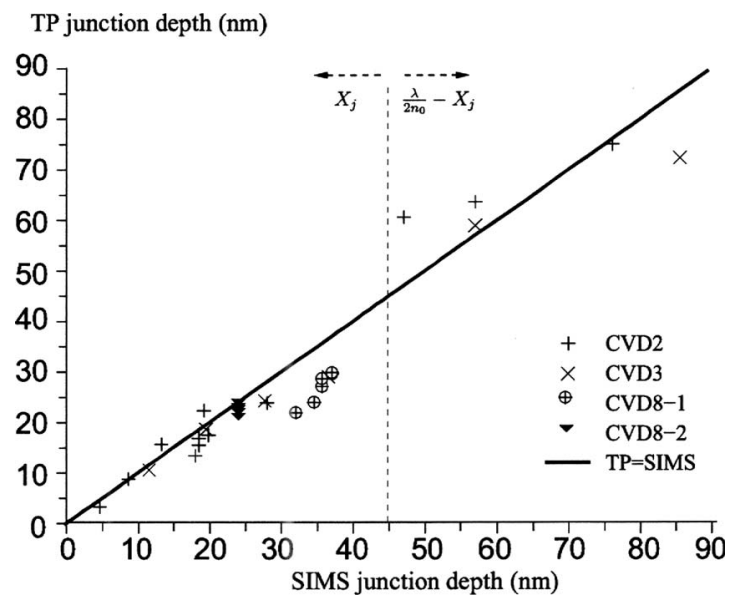

FIG. 2. Correlation between the SIMS junction depth of CVD boxlike profiles and the junction depth obtained using the described method. The thick black line shows the 1:1 correlation. On the left of the vertical dotted line $\left[X_{j}<\lambda /\left(4 n_{0}\right)=44 \mathrm{~nm}\right]$, formula (4) can be used as such. On the right of the vertical dotted line $\left[X_{j}>\lambda /\left(4 n_{0}\right)=44 \mathrm{~nm}\right]$, formula (4) gives $\left[\lambda /\left(2 n_{0}\right)-X_{j}\right]$.

For completeness, the technique has also been tested on the CVD5 and CVD4 matrices (see Table I of Ref. 12). However, the results are not accurate in either cases. In the case of CVD5, this is most likely due to the fact that the active doping is not high enough $\left(<5 \times 10^{18} \mathrm{~cm}^{-3}\right)$, so that $L_{\text {layer }}$ $\sim L_{\text {substrate }}{ }^{19}$ As for the CVD4, they contain a very high inactive doping concentration, making the substrate plasma component dependent on the doping profile. ${ }^{16}$

\section{B. Relative determination of junction depths}

Rather than using an undoped substrate as a reference like in formula (4), one can also use another doped layer with junction depth $X_{j}^{\text {reference }}$. In this case, the ratio of PMOR signals is

\section{a) Junction depth}

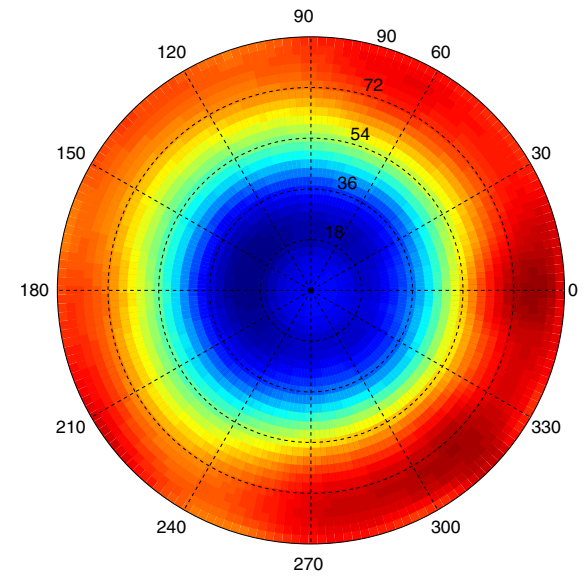

$$
\frac{\Delta R / R_{\text {layer }}^{\mathrm{I}}}{\Delta R / R_{\text {reference }}^{\mathrm{I}}} \rightarrow \frac{\cos \left(\frac{4 \pi n_{0} X_{j}}{\lambda}\right)}{\cos \left(\frac{4 \pi n_{0} X_{j}^{\text {reference }}}{\lambda}\right)} .
$$

The interest of formula (5) is that it gives a procedure for the relative determination of junction depths. In particular, if $X_{j}^{\text {reference }}<\lambda /\left(4 n_{0}\right)(\sim 44 \mathrm{~nm}$ for $\mathrm{TP}$ in $\mathrm{Si})$ and if $\Delta X_{j}=X_{j}$ $-X_{j}^{\text {reference }}$ is small, formula (5) gives after second-order Taylor expansion of the cosine in the numerator

$$
\begin{aligned}
\Delta X_{j}= & \frac{\lambda}{4 \pi n_{0}}\left\{-\tan \left(4 \pi n_{0} X_{j}^{\text {reference }} / \lambda\right)\right. \\
& +\operatorname{sign}\left(\tan \left(4 \pi n_{0} X_{j}^{\text {reference }} / \lambda\right)\right) \\
& \left.\times \sqrt{\tan ^{2}\left(4 \pi n_{0} X_{j}^{\text {reference }} / \lambda\right)+2\left(1-\frac{\Delta R / R_{\text {layer }}^{\mathrm{I}}}{\Delta R / R_{\text {reference }}^{\mathrm{I}}}\right)}\right\},
\end{aligned}
$$

where the sign function is, respectively, 1 or -1 according to whether its argument is positive or negative.

Performing the reference measurement at the center of a wafer, formula (6) can e.g., be used to determine the junction depth uniformity over a full wafer (e.g., using the powerful microuniformity mapping capabilities of $\mathrm{TP}^{21,22}$ ). An example is shown in Fig. 3(a) for an As-doped CVD layer. The reference is taken at the center with a nominal junction depth of $35 \mathrm{~nm}$. This 49-point junction depth map [Fig. 3(a)] is compared to a 973-point sheet resistance map [Fig. 3(b)] measured with a junction-photovoltage technique. These maps show a good agreement since the most resistive region (center of the wafer) is also the shallow junction region.

Notice that the technique cannot be used with a reference junction depth which would result in zero cosine (typically $22 \mathrm{~nm}$ in $\mathrm{Si}$ for the TP630XP). However, this is not a real physical limitation since one should just change the probe laser wavelength to solve this problem.
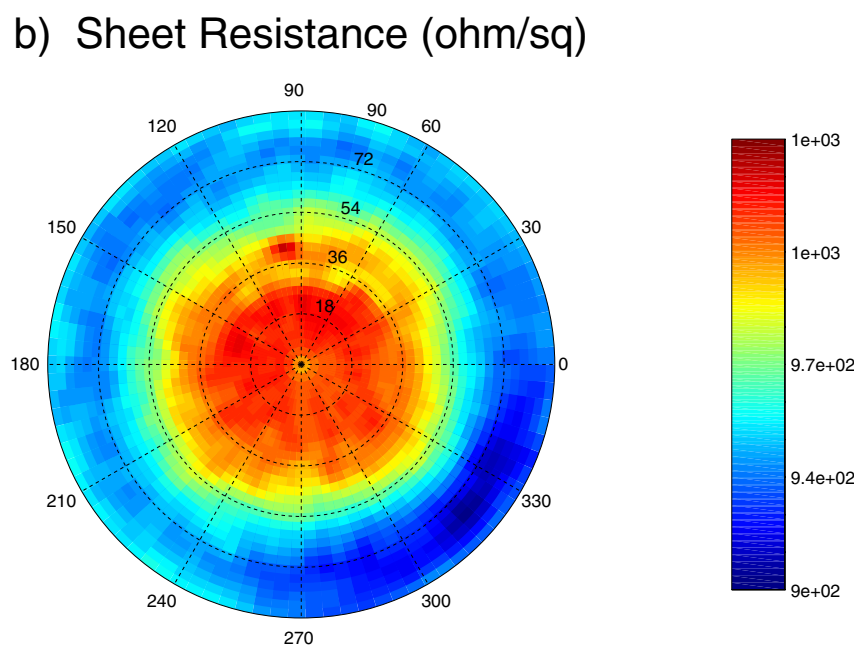

FIG. 3. (Color online) Wafer maps of (a) the junction depth and (b) the sheet resistance of a CVD-grown doped layer. 


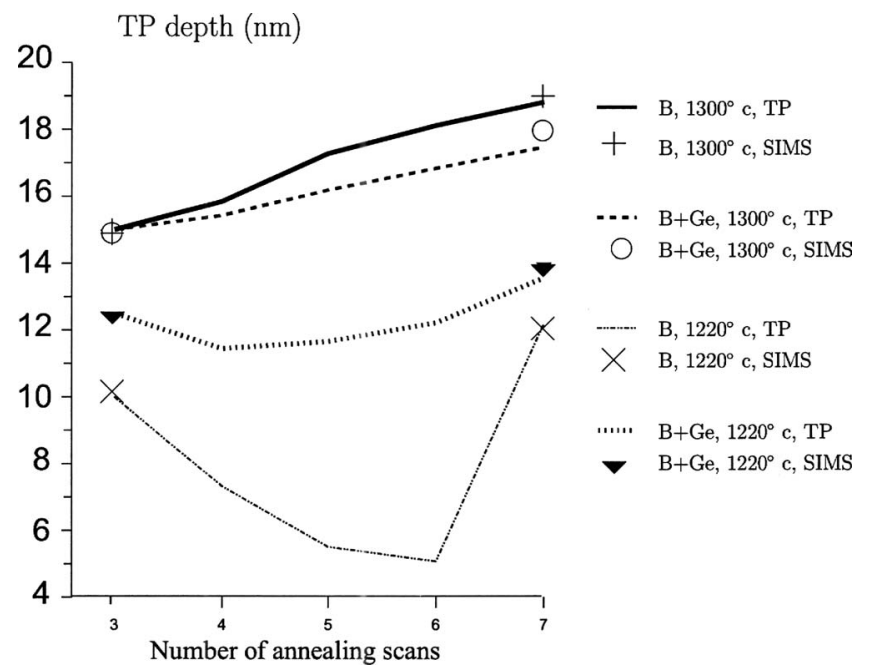

FIG. 4. TP depths (lines) and SIMS depths at $10^{20} \mathrm{~cm}^{-3}$ (symbols) as a function of the number of laser annealing scans for implanted samples. The TP depths obtained with the proposed procedure are in good agreement with the SIMS depths at $10^{20} \mathrm{~cm}^{-3}$ for the high annealing temperature.

\section{JUNCTION DEPTHS OF IMPLANTED PROFILES}

In this section, we discuss the possible extension of the proposed technique to arbitrarily shaped profiles. In particular, we focus on annealed implanted profiles. The samples for this study are described and fully characterized in Ref. 23. In summary, we study three series of samples implanted, respectively, with B only $\left(0.5 \mathrm{keV}, 10^{15} \mathrm{~cm}^{-2}\right)$, with B and Ge $\left(12 \mathrm{keV}, 5 \times 10^{14} \mathrm{~cm}^{-2}\right)$, or finally with $\mathrm{B}, \mathrm{Ge}$, and $\mathrm{C}$ $\left(3 \mathrm{keV}, 10^{15} \mathrm{~cm}^{-2}\right)$. All three series are then laser annealed with two different temperatures $\left(1220\right.$ and $\left.1300{ }^{\circ} \mathrm{C}\right)$ one to seven times, resulting in 14 different thermal budgets per series. Notice that each series was implanted on one single wafer, setting the perfect conditions for our method as the substrate signal is indeed more reproducible (see Sec. V).

The extension of the proposed technique to these implanted profiles brings essentially two problems. First, PMOR is not, strictly speaking, sensitive to the junction depth but to the depth where the profile slope is highest (reflection technique). The "TP depth" which this technique is able to determine can therefore be defined as the depth where the profile is the steepest. For the specific case of these implanted samples, the TP depth is the depth where the SIMS concentration is $10^{20} \mathrm{~cm}^{-3}$ (see Figs. 3 and 4 of Ref. 23). Second, a substrate signal being more difficult to define, formula (6) is preferred to Eq. (4). In this article, the samples annealed three times, usually assumed to be already well activated, are taken as references of each series and annealing temperature.

The obtained results are shown in Fig. 4 for the B implanted as well as the $\mathrm{B}+\mathrm{Ge}$ implanted samples. For the high annealing temperature, the obtained TP depth gives a very good agreement with the measured SIMS depths at $10^{20} \mathrm{~cm}^{-3}$. In particular, after seven scans, the TP depth and SIMS depth at $10^{20} \mathrm{~cm}^{-3}$ coincide for the two sets of samples. Besides, the TP depth shows a monotonic increase when increasing the number of annealing scans. For the low annealing temperature, the TP depth appears to show an unexpected nonmonotonic behavior. This cannot be attributed to any physical mechanisms. The samples are probably not sufficiently activated, in which case the presented theory fails. The last result to be reported is that the $\mathrm{C}+\mathrm{Ge}+\mathrm{B}$ implanted samples exhibit a purely thermal signal (too many remaining defects ${ }^{24}$ ) (similar behavior is also observed in Ref. 25). The proposed technique is based on the comparison of the substrate plasma component, this latter obviously needs to be dominant in order to yield useful results. However, these different behaviors are still very interesting because it implies that one is able to probe incomplete dissolution of defects in a highly activated profile.

In summary, this section shows that even though it is not designed for profiles of arbitrary shape, this technique can still be used on steep Gaussian type profiles and provide accurate results. Care should be taken in defining the measured depth, which is not the junction depth but rather the depth of highest profile slope. Similar to the case of boxlike active doping profiles, our technique can determine the relative TP depth or even the absolute TP depth provided that an equivalent substrate is available for reference. The technique works best in the case where most of the substrate defects have been annealed. The transition from thermal to plasma can be used as an indicator for the defect dissolutions.

\section{ACCURACY AND SENSITIVITY}

The accuracy of the technique depends on how well the two main assumptions made are satisfied. First, at a certain beam separation, the thermal and layer plasma components both should be negligible with respect to the substrate plasma component. When using TP on a silicon sample, the thermal component should cause no problem if there are few defects. Similarly, the layer plasma component can be considered to be small enough if the active doping is not too low $\left(>5 \times 10^{18} \mathrm{~cm}^{-3}\right)$.

Second, the lateral behavior of the substrate plasma component should be the same on the reference sample and on the test sample. This actually implies two different hypotheses. First, this postulates that the doping profiles themselves should have little impact on the substrate plasma component, which is confirmed by simulations as long as the active doping concentration is not too high $\left[<(1-2) \times 10^{20} \mathrm{~cm}^{-3}\right]$. The inactive doping concentration should also be as low as possible. The technique should therefore be preferentially used on well annealed structures. Second, it also means that all substrates need to be the same from the TP measurement point of view. To assess the validity of the second assumption, we measured offset curves on 20 different $n$-type and 20 different $p$-type subtrates (monitor wafers). While the PMOR signal variations on the $n$-type substrates are below $1 \%$, they reach up to $15 \%$ on the $p$-type substrates. Furthermore, the measurements show that the signal level on the $n$-type substrates is higher than that on the $p$-type substrates (up to $25 \%$ difference). This behavior is not expected in theory since the only relevant parameter is the high-injection 


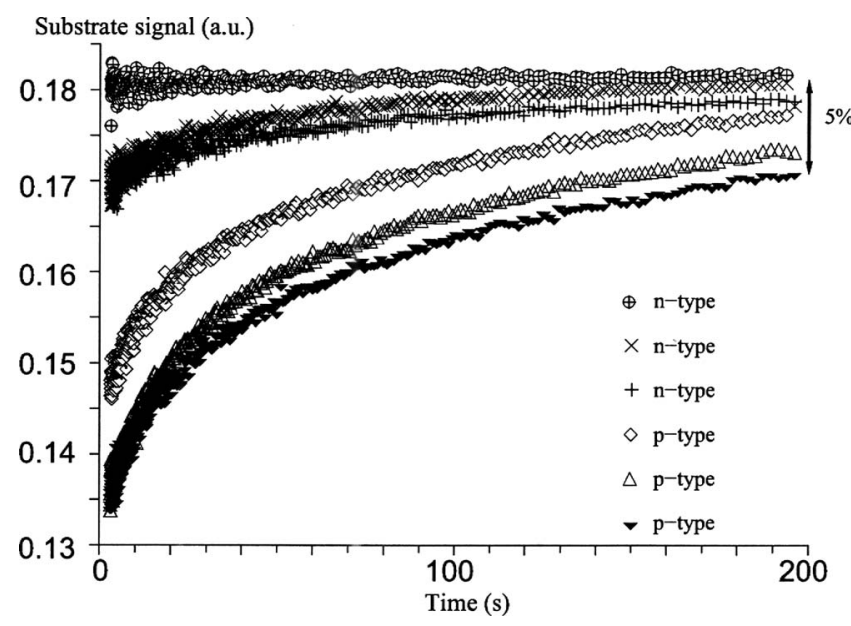

FIG. 5. Time dependence of the PMOR signal due to passivation of the surface on a series of $p$ - and $n$-type undoped substrates (doping type written at the bottom right of the figure). At saturation, the signals are expected to be the same for all substrates regardless of the doping type.

ambipolar diffusivity ${ }^{20}$ which should be independent of the doping type. Most of these differences can however be shown to be due to surface effects (Fig. 5). Indeed, if one records the signals as a function of time, carriers are injected in the surface and passivate it until the generated electric field between surface and bulk is too high for carriers to be further injected. ${ }^{19}$ This is observed experimentally as a saturation of the signal. It is at saturation that the signal is the least sensitive to the surface and is therefore more reproducible. Figure 5 shows that even though saturation is not reached on all substrates after $200 \mathrm{~s}$, the difference between the signals on these substrates, whether $n$ - or $p$-type, is only about 5\% (only a selection of curves is shown in Fig. 5). In conclusion, the technique will be more accurate if the surface is passivated.

Let us make three final remarks. First, even without surface passivation, the reproducibility of the substrate signal on different spots of the same wafer is about $1 \%$. The relative determination of the junction with respect to, e.g., the center point of a wafer would therefore not require charging (fast high-resolution mapping is feasible). Second, the wafers used in this article are not well characterized (monitor wafers). Measurements on well characterized high-quality device wafers show a wafer-to-wafer reproducibility below $0.1 \%$, which would make the technique much more accurate. Finally, to ensure that the assumptions are satisfied, full offset curves can be measured like in Fig. 1. The ratio of the signals $\Delta R / R_{\text {layer }}^{\mathrm{I}} / \Delta R / R_{\text {reference }}^{\mathrm{I}}$ should saturate at value between -1 and 1 when the beam spacing is large enough.

The sensitivity of the technique can be calculated by measuring the signal reproducibility on various undoped substrates. Here we use the 5\% reproducibility obtained after surface charging (see Fig. 5) on both $n$-type and $p$-type substrates (worst case scenario). The first derivative of formula (3) with respect to the junction depth gives

$$
\begin{aligned}
\Delta X_{j} & =\left|\frac{\partial X_{j}}{\partial \Delta R / R}\right| \Delta(\Delta R / R) \\
& =\frac{1}{\left|\frac{\partial \Delta R / R}{\partial X_{j}}\right| \Delta(\Delta R / R)} \\
& =\frac{\lambda}{4 \pi n_{0}}\left|\cot \left(\frac{4 \pi n_{0} X_{j}}{\lambda}\right)\right| \frac{\Delta(\Delta R / R)}{\Delta R / R_{\text {substrate }}} \\
& \leqslant 0.75\left|\cot \left(\frac{4 \pi n_{0} X_{j}}{\lambda}\right)\right| .
\end{aligned}
$$

The technique has therefore a subnanometer sensitivity for junction depths between 10 and $35 \mathrm{~nm}$ with the current TP630XP system. These values include both the tool and wafer-to-wafer reproducibility.

\section{CONCLUSION}

In this article, we presented a technique for determining the junction depth of an active doping profile from PMOR measurements. Though this procedure is based on our physical understanding, no modeling is required during its application. This is due to the fact that, at large beam offsets, the PMOR signal is mainly determined by the junction depth. The ratio of the PMOR signal measured on the unknown sample just needs to be divided by the signal measured on a reference sample. If the reference is an undoped substrate, the technique offers the absolute junction depth through formula (4). If the reference is chosen to be another doped layer, formula (5) or (6) help determine the relative junction depth. The technique works on CVD boxlike profiles if the active doping is high enough and the inactive doping concentration is low enough and on implanted layers if the defect density is not significant. Experiments show a very good correlation with SIMS on ultrashallow profiles $(<30 \mathrm{~nm})$. On implanted profiles, the obtained depth is the depth at which the profile is the steepest and shows a very good agreement with SIMS on well activated samples. Finally, we have shown that a good monitoring of the substrate PMOR signal is essential for the technique to be accurate and reproducible. By passivating the surface (e.g., by a longer measurement), a sub-nm depth sensitivity for junction depths between 10 and $35 \mathrm{~nm}$ is achievable with a probe wavelength of $670 \mathrm{~nm}$.

\section{ACKNOWLEDGMENTS}

This research was funded by a Ph.D. grant of the Institute for the Promotion of Innovation through Science and Technology in Flanders (IWT-Vlaanderen). The authors would like to thank Jozefien Goossens, Joris Delmotte, and Luc Geenen (IMEC) for the SIMS measurements.

\footnotetext{
${ }^{1}$ International Technology Roadmap for Semiconductors (http:// Www.itrs.net).

${ }^{2}$ W. Vandervorst, T. Janssens, C. Huyghebaert, and B. Berghmans, Appl. Surf. Sci. 255, 1206 (2008).

${ }^{3}$ T. Clarysse, D. Vanhaeren, I. Hoflijk, and W. Vandervorst, Mater. Sci. Eng. R. 47, 123 (2004).

${ }^{4}$ R. E. Wagner and A. Mandelis, J. Phys. Chem. Solids 52, 1061 (1991).
} 
${ }^{5}$ J. Opsal, M. W. Taylor, W. L. Smith, and A. Rosencwaig, J. Appl. Phys. 61, 240 (1987)

${ }^{6}$ A. Rosencwaig, J. Opsal, W. L. Smith, and D. Willenborg, Appl. Phys. Lett. 46, 1013 (1985).

${ }^{7}$ J. Opsal and A. Rosencwaig, Appl. Phys. Lett. 47, 498 (1985).

${ }^{8}$ A. Mandelis and R. E. Wagner, Jpn. J. Appl. Phys., Part 1 35, 1786 (1996).

${ }^{9}$ C. Christofides, I. A. Vitkin, and A. Mandelis, J. Appl. Phys. 67, 2815 (1990).

${ }^{10}$ M. Fox, Optical Properties of Solids (Oxford University Press, Oxford, 2001).

${ }^{11}$ G. E. Jellison, Jr. and F. A. Modine, J. Appl. Phys. 76, 3758 (1994).

${ }^{12}$ J. Bogdanowicz, F. Dortu, T. Clarysse, W. Vandervorst, D. Shaughnessy, A. Salnik, L. Nicolaides, and J. Opsal, J. Vac. Sci. Technol. B 26, 310 (2008).

${ }^{13}$ A. Salnick and J. Opsal, J. Appl. Phys. 91, 2874 (2002).

${ }^{14}$ T. Clarysse, W. Vandervorst, M. Bakshi, L. Nicolaides, A. Salnik, and J. Opsal, J. Vac. Sci. Technol. B 24, 1139 (2006).

${ }^{15}$ L. Nicolaides, A. Salnick, and J. Opsal, Rev. Sci. Instrum. 74, 586 (2003).

${ }^{16}$ J. Bogdanowicz, F. Dortu, T. Clarysse, W. Vandervorst, D. Shaughnessy,
A. Salnik, and L. Nicolaides, Mater. Sci. Eng., B 154-155, 234 (2008).

${ }^{17}$ J. Bogdanowicz, F. Dortu, T. Clarysse, W. Vandervorst, D. Shaughnessy, and A. Salnik, J. Appl. Phys. (to be published).

${ }^{18}$ A. Mandelis, Diffusion-Wave Fields: Mathematical Methods and Green Functions, 1st ed. (Springer, New York, 2001).

${ }^{19}$ F. Dortu, Ph.D. thesis, Katholieke Universiteit Leuven, 2009.

${ }^{20}$ F. Dortu, T. Clarysse, R. Loo, B. Pawlak, R. Delhougne, and W. Vandervorst, J. Vac. Sci. Technol. B 24, 1131 (2006).

${ }^{21}$ J. Borland, IEEE International Conference on Advanced Thermal Processing of Semiconductors, 2007 (unpublished).

${ }^{22}$ E. Rosseel, J. Bogdanowicz, T. Clarysse, W. Vandervorst, A. Salnik, and L. Nicolaides, Proceedings of the Insight Workshop, 2009 (unpublished).

${ }^{23} \mathrm{E}$. Rosseel et al., IEEE International Conference on Advanced Thermal Processing of Semiconductors, 2008 (unpublished).

${ }^{24}$ W. Vandervorst, T. Clarysse, B. Brijs, R. Loo, Y. Peytier, B. Pawlak, E. Budiarto, and P. Borden, 2003 International Conference on Characterization and Metrology for ULSI Technology [AIP Conf. Proc. 683, 758 (2003)].

${ }^{25}$ T. Clarysse et al., Proceedings of the Insight Workshop, 2009 (unpublished). 